\title{
Faecal detection of the lungworms Crenosoma vulpis and Angiostrongylus vasorum and serological detection of $A$. vasorum in dogs from the Czech Republic
}

\author{
Markéta Hajnalová ${ }^{1}$, Vlasta Svobodová1, Manuela Schnyder ${ }^{2}$, Roland Schaper $^{3}$, \\ Miroslav Svoboda ${ }^{4}$

\begin{abstract}
${ }^{1}$ University of Veterinary and Pharmaceutical Sciences Brno, Faculty of Veterinary Medicine, Department of Pathological Morphology and Parasitology, Brno, Czech Republic

${ }^{2}$ University of Zurich, Vetsuisse Faculty, Institute of Parasitology, Zurich, Switzerland

${ }^{3}$ Bayer Animal Health GmbH, Leverkusen, Germany

${ }^{4}$ University of Veterinary and Pharmaceutical Sciences Brno, Faculty of Veterinary Medicine, Small Animal Clinic, Brno, Czech Republic
\end{abstract}

Received March 8, 2017

Accepted December 19, 2017

\begin{abstract}
Dogs are definitive hosts for the lungworms Crenosoma vulpis and Angiostrongylus vasorum. Intermediate hosts are gasteropoda (Mollusca). In the past, lungworms in dogs were rarely identified in the Czech Republic and in neighbouring countries. A total of 253 dogs were sampled for faecal analysis performed by the Baermann method for isolation of first stage larvae (L1) of lungworms. A total of 193 serum samples were collected and analysed by ELISA for the detection of a circulating antigen and specific antibodies against $A$. vasorum. All dog owners were asked to fill out a questionnaire. Nine dogs $(4.7 \%, 95 \%$ Confidence Interval, CI: $2.2-8.7 \%)$ were positive for the A. vasorum circulating antigen, seven dogs (3.6\%, CI: $1.5-7.3 \%)$ had specific antibodies against $A$. vasorum; of these, three animals (1.6\%, CI: $0.3-4.5 \%)$ were positive for both ELISAs. Most of them were under the age of three years. Only three of 13 seropositive dogs were not healthy, namely, in one dog vomitus was observed and two dogs were pyretic. Faecal samples of 5 dogs were positive for C. vulpis L1 (2.0\%, CI: 0.6-4.6\%). Angiostrongylus vasorum L1 were found in the faecal sample of one $\operatorname{dog}(0.4 \%, \mathrm{CI}: 0.01-2.2 \%)$. This study illustrates the wide-spread occurrence of lungworms in the Czech Republic. Disease awareness of lungworms among animal owners and veterinarians should be implemented.
\end{abstract}

Dog, lungworms, coproscopy, serology, Crenosoma vulpis, Angiostrongylus vasorum

In the past, lungworms in dogs were rarely identified in the Czech Republic and the neighbouring countries, and therefore, not addressed in research purposes or in differential diagnosis of respiratory diseases, only to become more known in the last five years. Dogs and wild Canidae are definitive hosts of the most common nematode lungworms Crenosoma vulpis and Angiostrongylus vasorum. Intermediate hosts include various species of gasteropoda (Mollusca) for both parasites (Guilhon and Bressou 1960; Stockdale and Hulland 1970), while frogs are described as paratenic hosts for A. vasorum (Bolt et al. 1993). Adult C. vulpis nematodes are located in the bronchi causing bronchitis and bronchopneumonia, frequently accompanied by coughing (Unterer et al. 2002). Its occurrence is widely known since the 1970s (Stockdale and Hulland 1970), particularly in red foxes (Willingham et al. 1996; Rajkovic-Janje et al. 2002; Jeffery et al. 2004), which are also considered to be an important wild reservoir for $A$. vasorum (Jeffery et al. 2004; Taylor et al. 2015; Tolnai et al. 2015). However, only in the last two decades, infections with this latter parasite, residing in the heart and pulmonary arteries in the adult stage, have been recorded in a massively increasing number of European countries out from the traditional endemic foci in France (Guilhon 1963) or Ireland (Dodd 1973). Very recent reports from Eastern European countries describe the presence of the parasite

Address for correspondence:

Prof. MVDr. Vlasta Svobodová, PhD, DipEVPC

Department of Pathological Morphology and Parasitology

Faculty of Veterinary Medicine UVPS Brno

Palackého tř. 1946/1

Phone: +420 541562265

612 42, Brno, Czech Republic

http://actavet.vfu.cz/ 
in Hungary (Schnyder et al. 2015b; Tolnai et al. 2015), Croatia (Rajkovic-Janje et al. 2002) and also from countries surrounding the Czech Republic. Germany, bordering northwesterly, is the one with long-standing reports of A. vasorum cases (Pallaske 1967; Eckert and Lämmler 1972) and several epidemiological studies confirmed its presence in particular in the south-eastern part of Germany (Taubert et al. 2008; Barutzki and Schaper 2009; Schnyder et al. 2013a). Further recent studies report A. vasorum from Poland (Schnyder et al. 2013b; Demiaszkiewicz et al. 2014), bordering northerly to the Czech Republic, and from Slovakia (Hurnikova et al. 2013; Miterpakova et al. 2015), bordering easterly.

Canine angiostrongylosis may cause severe or even fatal disease, frequently manifesting through severe respiratory signs and coagulopathies and occasionally through neurological signs (Garosi et al. 2005; Koch and Willesen 2009; Gredal et al. 2011). However, infections may also pass unnoticed when subclinical, or are misdiagnosed because clinical signs are so varied and/or non-specific. Therefore, the use of appropriate diagnostic procedures is of fundamental importance, together with disease awareness. Among the procedures for parasite detection, the Baermann method (Deplazes et al. 2016) performed with faecal samples possibly collected over three consecutive days is recommended for the isolation of first stage larvae (L1), which can be observed microscopically. These need to be distinguished from other larvae present in faeces according to morphological characters. The need for rapid and accurate diagnosis has led to the development and introduction of serological tests to detect $A$. vasorum antigen (Schnyder et al. 2011) and antibodies (Schucan et al. 2012), recommended for individual diagnosis and for mass-screening as well. These ELISAs showed high sensitivity and specificity compared with analysis of faecal samples and biomolecular procedures (Schnyder et al. 2015a).

The aim of our study was to determine the occurrence of C. vulpis and A. vasorum infections in dogs from the Czech Republic adopting the Baermann method for isolation of lung worm larvae, and to perform serological tests for the detection of A. vasorum in dogs.

\section{Materials and Methods}

A total of 28 veterinary clinics from different locations in the Czech Republic participated in the study. From April to September 2013, a total of 253 dogs aged 2 months to 15 years were investigated: faecal samples were obtained from all dogs; from 193 dogs also blood samples were collected. Dogs were included in the study unless they were administered macrocyclic lactones for at least 3 months before sampling. Serum was maintained at $-20{ }^{\circ} \mathrm{C}$ until processed at the Institute of Parasitology, University of Zurich. Faecal samples were examined within three days or were kept at $-20{ }^{\circ} \mathrm{C}$ until processed.

Dog owners involved in the monitoring completed a questionnaire regarding the sex, age, outdoor activities of the dogs and clinical signs associated with the impaired respiratory tract (cough, fever, loss of appetite, running nose, etc.).

\section{Serological analyses}

The serum samples were analysed with ELISAs for the detection of circulating antigens of $A$. vasorum (Schnyder et al. 2011; sensitivity 95.7\%, specificity 94.0\%) and for the detection of specific antibodies against A. vasorum (Schucan et al. 2012; sensitivity $85.7 \%$, specificity $98.8 \%$ ). Each plate was run with two positive controls (sera from experimentally infected dogs), two negative controls (from uninfected laboratory dogs) and a conjugate control. A diluted positive serum was added twice on each plate to calculate a correction factor for adjustment between plates, as previously described (Schnyder et al. 2011). The cut-off value was calculated as mean plus three standard deviations of randomly selected sera.

Analysis of faeces

The faecal samples were examined using the Baermann method (Kassai 1999) for isolation of L1, which were identified after microscopic observation. A single sample was collected from each dog.

Statistical analyses

Excel 2007 for Windows (Microsoft Corporation, Redmond USA) was used to calculate proportions and $95 \%$ confidence intervals $(\mathrm{CI})$. 


\section{Results}

\section{Serological results}

From 193 serum samples, a total of nine dogs (4.7\%, CI: $2.2-8.7 \%)$ were positive for the A. vasorum circulating antigen, and in seven dogs (3.6\%, CI: $1.5-7.3 \%)$ specific antibodies against $A$. vasorum were detected; three animals $(1.6 \%, \mathrm{CI}: 0.3-4.5 \%)$ were positive for both ELISAs. Serological positive results were therefore detected in a total of $13(6.7 \%)$ of the 193 animals tested. Sex, age, and lifestyle including the dog walking regime in a group of 180 negative and 13 positive animals are compared in Table 1 . Positive individuals were aged 8 months to 12 years, 7 were males and 6 females. Most of them $(n=10$, five males and five females) were under the age of three years, while the remaining three seropositive animals were older ( 2 males, 1 female). Lifestyle and outdoor activities were similar regardless of serological results. Table 2 summarises owner observations and results of clinical examination in both groups. Only three of 13 seropositive dogs were not healthy, namely, in one dog vomitus was observed and two dogs were pyretic.

Table 1. General profile of the tested dogs based on the owner's questionnaire $(n=193)$.

\begin{tabular}{|c|c|c|c|c|}
\hline \multirow[t]{2}{*}{ Sex/Age/Lifestyle/Walking } & \multicolumn{2}{|c|}{$\begin{array}{l}\text { Seronegative } \\
\quad(\mathrm{n}=180)\end{array}$} & \multicolumn{2}{|c|}{$\begin{array}{l}\text { Seropositive } \\
\quad(\mathrm{n}=13)\end{array}$} \\
\hline & No & $\%$ & No & $\%$ \\
\hline Intact male & 68 & 37.8 & 5 & 38.5 \\
\hline Castrated male & 13 & 7.2 & 2 & 15.4 \\
\hline Intact female & 74 & 41.1 & 5 & 38.5 \\
\hline Neutered female & 25 & 13.9 & 1 & 7.6 \\
\hline In total & 180 & 100 & 13 & 100 \\
\hline Age under one year & 16 & 8.9 & 2 & 15.4 \\
\hline Age one to 3 years & 56 & 31.1 & 8 & 61.5 \\
\hline Age 3.1 to 8 years & 93 & 51.7 & 2 & 15.4 \\
\hline Older than 8 years & 15 & 8.3 & 1 & 7.7 \\
\hline In total & 180 & 100 & 13 & 100 \\
\hline Dogs living indoor & 116 & 64.4 & 8 & 61.5 \\
\hline Dogs living outdoor & 64 & 35.6 & 5 & 38.5 \\
\hline In total & 180 & 100 & 13 & 100 \\
\hline Walking outside less than $1 \mathrm{~h}$ a day & 15 & 8.3 & 1 & 7.7 \\
\hline Walking outside one to $2 \mathrm{~h}$ a day & 31 & 17.2 & 1 & 7.7 \\
\hline Walking/running outside more than $2 \mathrm{~h}$ a day & 134 & 74.5 & 11 & 84.6 \\
\hline In total & 180 & 100 & 13 & 100 \\
\hline Walking on a leash & 21 & 11.7 & 2 & 15.4 \\
\hline Free walking/running & 68 & 37.8 & 4 & 30.8 \\
\hline Combination (leash walking and free running) & 91 & 50.5 & 7 & 53.8 \\
\hline In total & 180 & 100 & 13 & 100 \\
\hline
\end{tabular}

\section{Results of copromicroscopical faecal analyses}

In total, faecal samples of 5 dogs were positive for $C$. vulpis L1 (2.0\%, CI: 0.6-4.6\%). These were one intact male dog aged 6 months, three dogs aged 3 years (two neutered females and one intact male) and one intact male aged 6 years. The owner observed prolonged cough in the one dog (intact male) aged 3 years, the other dogs were without clinical signs. Angiostrongylus vasorum L1 were found in the faecal sample of one dog $(0.4 \%$, CI: $0.01-2.2 \%)$. It was an intact female aged one year, in which no clinical signs were observed. This animal was not tested by ELISA as no serum was available. 
Table 2. Comparison of the clinical symptomatology of $A$. vasorum seronegative $(\mathrm{n}=180)$ and seropositive $(\mathrm{n}=13)$ dogs.

\begin{tabular}{lrrrr}
\hline Owner's observations & \multicolumn{2}{c}{$\begin{array}{c}\text { Seronegative } \\
(\mathrm{n}=180)\end{array}$} & \multicolumn{2}{c}{$\begin{array}{c}\text { Seropositive } \\
(\mathrm{n}=13)\end{array}$} \\
\cline { 2 - 5 } Results of clinical examination & No & Per cent & No & Per cent \\
\hline Without symptoms of any disease (healthy dogs) & 153 & 85.0 & 10 & 76.9 \\
Observations/Clinical signs & 27 & 15.0 & 3 & 23.0 \\
Loss of weight & 2 & 1.1 & 0 & 0 \\
Anorexia & 2 & 1.1 & 0 & 0 \\
Fever & 4 & 2.2 & 2 & 15.4 \\
Running nose & 4 & 2.2 & 0 & 0 \\
Cough & 12 & 6.7 & 0 & 0 \\
Vomiting & 0 & 0 & 1 & 7.7 \\
Diarrhoea & 3 & 1.7 & 0 & 0 \\
\hline In total & 180 & 100 & 13 & 100 \\
\hline
\end{tabular}

\section{Discussion}

Our study showed that both lungworms, C. vulpis and A. vasorum, occur in the canine population of the Czech Republic. With a prevalence of $2.0 \%$ (CI: $0.6-4.6 \%$ ) for C. vulpis, these results are comparable with studies done in the neighbouring German Federal state of Bavaria (0.36-13.4\%), (Barutzki and Schaper 2009; Barutzki 2013; Schulz et al. 2013) and higher than in the Federal state of Sax ony (0.25\%), (Barutzki and Schaper 2009). Crenosoma vulpis infection was reported only once in the Czech Republic in a one-yearold Shetland Sheepdog presented with chronic cough (Husník et al. 2011). The prevalence for $A$. vasorum obtained by copromicroscopic analysis was lower $(0.4 \%, \mathrm{CI}$ : $0.01-2.2 \%)$, but non-significantly, compared to $C$. vulpis. In opposition to that, serology revealed nine $A$. vasorum antigen positive dogs, specific antibodies were detected in seven dogs and both tests were positive in three animals. Overall, we found at least one positive result of serological response in 13 dogs $(6.7 \%)$. Serological results identified therefore more positive dogs than coproscopy, confirming higher sensitivity of the adopted serological methods (Guardone et al. 2013; Schnyder etal.2015a). However, the interpretation of results obtained with the two ELISAs is different: antigen detection represents an actual infection, while specific antibody detection stands for parasite exposure. Furthermore, false positive results and cross-reactions cannot be completely excluded. The results with the highest positive predictive value are the dogs being positive with both ELISAs (Schnyder etal. 2013a). Interestingly, the here observed prevalence of $1.6 \%$ (CI: $0.3-4.5 \%$ ) for these latter dogs is comparable or higher than that in the countries where the presence of $A$. vasorum was discovered decades ago, such as Germany (1.2\% determined by faecal analysis) (Taubert et al. 2008), England (0.97\%) (Schnyder et al. 2013a) or Italy $(0.8-0.9 \%)$ (Guardone et al. 2013). This could indicate that the parasite has been present for some time without dog owners and veterinarians being aware of it. In fact, Baermann analysis is not routinely performed, and a serological in-clinic assay (Angio Detect ${ }^{\mathrm{TM}}$ Test, IDEXX Laboratories, Westbrook, Maine, USA) for the detection of $A$. vasorum antigen has been available commercially only recently. The broad spectrum of clinical signs including asymptomatic dogs and a slow progression of the disease make the diagnosis of dogs infected with $A$. vasorum challenging. Together with limited disease awareness, canine angiostrongylosis can be easily missed.

Also the recent reports of $A$. vasorum in dogs and foxes from countries surrounding the Czech Republic (Germany, Poland, Slovakia) sustain the hypothesis that $A$. vasorum may have been established for some time in the Czech Republic. Further investigations within the fox population acting as a potential and highly suitable reservoir of the parasite would help to clarify the situation as well as how successful $A$. vasorum was or will be in the future in spreading in the Czech Republic. 
Faecal sampling during only one day for larvoscopy was necessary due to practical reasons, and multiple samples could not be obtained. Furthermore, as the study was performed in 28 veterinary practices distributed across the Czech Republic, it was necessary to keep 106 faecal samples frozen at $-20^{\circ} \mathrm{C}$ until examination in our laboratory. Both the freezing and the single day faecal samples may have influenced the outcome of the results with high probability, leading to lower detection rates, although the ability of the genus Crenosoma L1 to resist freezing temperatures for varying lengths of time has been described (Snyder 1985). The larvae were still motile even after having been frozen at $-25^{\circ} \mathrm{C}$ for periods of up to 14 months. It was supported also by other studies in which survival of the L1 of C. vulpis under freezing temperatures was observed (Saeed et al. 2006). Angiostrongylus vasorum $\mathrm{L} 1$ were suggested to not be able to survive temperatures below $-20{ }^{\circ} \mathrm{C}$ for more than $24 \mathrm{~h}$ (Jeffery et al. 2004). For both, C. vulpis and A. vasorum L1 detection, it has to be assumed that sensitivity using copromicroscopic methods with frozen samples was decreased.

An identified potential risk factor for positive results was the age (higher risk for younger dogs). The breed, neutering status, and sex were not relevant risk factors. The same results were obtained by Morgan et al. (2010). Most of the dogs in our study positive for $A$. vasorum or $C$. vulpis were individuals aged up to three years, but the infection affected older dogs, too, including a 12-year-old senior dog. The majority of the dogs had opportunities to run free daily and to sniff in the grass or play with sticks (Schnyder 2015), and therefore to occasionally lick or ingest snails as intermediate hosts, which is a prerogative for infection with lungworms. Especially young dogs may be attracted by snails or slugs. The majority of positive dogs in our study did not show clinical signs, but single individuals with fever and respiratory problems occurred as well.

Dog lungworms can cause serious diseases (Kruse et al. 2013) and therefore, the diagnosis of lungworms should be included in the differential diagnosis of pyrexia and respiratory problems. Particularly in areas where lungworms are not routinely searched for, disease awareness among animal owners and veterinarians should be implemented.

\section{Acknowledgements}

We would like to express many thanks to our colleagues from 28 veterinary practices who examined the dogs and collected blood samples as well as to the dog owners who obtained faeces and completed our questionary.

\section{References}

Barutzki D 2013: Nematode infections of the respiratory tract in dogs in Germany. Tierarztl Prax Ausg K Kleintiere Heimtiere 41: 326-336

Barutzki D, Schaper R 2009: Natural infections of Angiostrongylus vasorum and Crenosoma vulpis in dogs in Germany (2007-2009). Parasitol Res 105 (Suppl 1): S39-S48

Bolt G, Monrad J, Frandsen F, Henriksen P, Dietz HH 1993: The common frog (Rana temporaria) as a potential paratenic and intermediate host for Angiostrongylus vasorum. Parasitol Res 79: 428-430

Demiaszkiewicz AW, Pyziel AM, Kuligowska I, Lachowicz J 2014: The first report of Angiostrongylus vasorum (Nematoda; Metastrongyloidea) in Poland, in red foxes (Vulpes vulpes). Acta Parasitol 59: 758-762

Deplazes P, Eckert J, Mathis A, von Samson-Himmelstjerna G, Zahner H 2016: Parasitology in Veterinary Medicine (Wageningen, Wageningen Academic Publishers).

Dodd K 1973: Angiostrongylus vasorum (Baillet, 1866) infestation in a greyhound kennels. Vet Rec 92: 195-197

Eckert J, Lämmler G 1972: Angiostrongylose bei Mensch und Tier. Z Parasitenk 39: 303-322

Garosi LS, Platt SR, McConnell JF, Wrayt JD, Smith KC 2005: Intracranial haemorrhage associated with Angiostrongylus vasorum infection in three dogs. J Small Anim Pract 46: 93-99

Gredal H, Willesen JL, Jensen HE, Nielsen OL, Kristensen AT, Koch J, Kirk RK, Pors SE, Skerritt GC, Berendt M 2011: Acute neurological signs as the predominant clinical manifestation in four dogs with Angiostrongylus vasorum infections in Denmark. Acta Vet Scand 53: 43

Guardone L, Schnyder M, Macchioni F, Deplazes P, Magi M 2013: Serological detection of circulating Angiostrongylus vasorum antigen and specific antibodies in dogs from central and northern Italy. Vet Parasitol 192: 192-198 
Guilhon J 1963: Recherches sur le cycle évolutif du Strongle des vaisseaux du chien. Bull Acad Vét 36: 431-442

Guilhon J, Bressou C 1960: Rôle des Limacidés dans le cycle évolutif d'Angiostrongylus vasorum (Baillet, 1866). C R Acad Sc 251: 2252-2253

Hurnikova Z, Miterpakova M, Mandelik R 2013: First autochthonous case of canine Angiostrongylus vasorum in Slovakia. Parasitol Res 112: 3505-3508

Husnik R, Sloboda M, Kovarikova S, Koudela B 2011: Infection with Crenosoma vulpis lungworm in a dog in the Czech Republic. Helminthologia 48: 56-58

Jeffery RA, Lankester MW, McGrath MJ, Whitney HG 2004: Angiostrongylus vasorum and Crenosoma vulpis in red foxes (Vulpes vulpes) in Newfoundland, Canada. Can J Zool 82: 66-74

Kassai T 1999: Veterinary Helminthology, Butterworth Heinemann, London, ISBN 0750635630

Koch J, Willesen JL 2009: Canine pulmonary angiostrongylosis: An update. Vet J 179: 348-359

Kruse BD, Hartmann K, Groth A, Schulz B, Wehner A 2013: Disseminated intravascular coagulopathy in a dog with angiostrongylus vasorum infection. Tierarztl Prax Ausg K Kleintiere Heimtiere 41: 401-407

Miterpakova M, Schnyder M, Schaper R, Hurnikova Z, Cabanova V 2015: Serological survey for canine angiostrongylosis in Slovakia. Helminthologia 52: 205-210

Morgan ER, Jefferies R, van Otterdijk L, McEniry RB, Allen F, Bakewell M, Shaw SE 2010: Angiostrongylus vasorum infection in dogs: Presentation and risk factors. Vet Parasitol 173: 255-261

Pallaske G 1967: Zur Angiostrongylose des Hundes. Dtsch Tierarztl Wochenschr 74: 166-171

Rajkovic-Janje R, Marinculic A, Bosnic S, Benic M, Vinkovic B, Mihaljevic Z 2002: Prevalence and seasonal distribution of helminth parasites in red foxes (Vulpes vulpes) from the Zagreb County (Croatia). Z Jagdwiss 48: $151-160$

Saeed I, Maddon-Hyttel C, Monrad J, Kapel CMO 2006: Helminths of red foxes (Vulpes vulpes) in Denmark. Vet Parasitol 139: 168-179

Schulz BZ, Seybold N, Sauter-Louis C, Hartmann K 2013: Prevalence of Angiostrongylus vasorum and Crenosoma vulpis in dogs in Bavaria. Berl Munch Tierarztl Wochenschr 71: 62-68

Schnyder M, Tanner I, Webster P, Barutzki D, Deplazes P 2011: An ELISA for sensitive and specific detection of circulating antigen of Angiostrongylus vasorum in serum samples of naturally and experimentally infected dogs. Vet Parasitol 179:152-158

Schnyder M, Schaper R, Bilbrough G, Morgan ER, Deplazes P 2013a: Seroepidemiological survey for canine angiostrongylosis in dogs from Germany and the UK using combined detection of Angiostrongylus vasorum antigen and specific antibodies. Parasitol 140: 1442-1450

Schnyder M, Schaper R, Pantchev N, Kowalska D, Szwedko A, Deplazes P 2013b: Serological detection of circulating Angiostrongylus vasorum antigen- and parasite-specific antibodies in dogs from Poland. Parasitol Res 112 (Suppl 1): 109-117

Schnyder M 2015: Slugs and Angiostrongylus vasorum - how much do we know? The Vet Rec 177: 44-45

Schnyder M, Jefferies R, Schucan A, Morgan ER, Deplazes P 2015a: Comparison of coprological, immunological and molecular methods for the detection of dogs infected with Angiostrongylus vasorum before and after anthelmintic treatment. Parasitol 142: 1270-1277

Schnyder M, Schaper R, Lukacs Z, Hornok S, Farkas R 2015b: Combined serological detection of circulating Angiostrongylus vasorum antigen and parasite-specific antibodies in dogs from Hungary. Parasitol Res $\mathbf{1 1 4}$ (Suppl 1) S145-S154

Schucan A, Schnyder M, Tanner I, Barutzki D, Traversa D, Deplazes P 2012: Detection of specific antibodies in dogs infected with Angiostrongylus vasorum. Vet Parasitol 185: 216-224

Snyder D 1985: The variability of $1^{\text {st }}$-stage Crenosoma goblei (Nematoda, Metastrongyloidea) larvae at $-25^{\circ} \mathrm{C}$. J Parasitol 71: 386-387

Stockdale PH, Hulland TJ 1970: The pathogenesis route of migration, and development of Crenosoma vulpis in the dog. Pathol Vet 7: 28-42

Taubert A, Pantchev N, Vrhovec MG, Bauer C, Hermosilla C 2008: Lungworm infections (Angiostrongylus vasorum, Crenosoma vulpis, Aelurostrongylus abstrusus) in dogs and cats in Germany and Denmark in 2003-2007. Vet Parasitol 159: 175-180

Taylor CS, Garcia Gato R, Learmount J, Aziz NA, Montgomery C, Rose H, Coulthwaite CL, McGarry JW, Forman DW, Allen S, Wall R, Morgan ER 2015: Increased prevalence and geographic spread of the cardiopulmonary nematode Angiostrongylus vasorum in fox populations in Great Britain. Parasitol 142: 1190-1195

Tolnai Z, Szell Z, Sreter T 2015: Environmental determinants of the spatial distribution of Angiostrongylus vasorum, Crenosoma vulpis and Eucoleus aerophilus in Hungary. Vet Parasitol 207: 355-358

Unterer S, Deplazes P, Arnold P, Flückiger M, Reusch CE, Glaus TM 2002: Spontaneous Crenosoma vulpis infection in 10 dogs: laboratory, radiographic and endoscopic findings. Schweiz Arch für Tierheilkd 144: $174-179$

Willingham AL, Ockens NW, Kapel CM, Monrad J 1996: A helminthological survey of wild red foxes (Vulpes vulpes) from the metropolitan area of Copenhagen. J Helminthol 70: 259-263 